\title{
Design of an Audience Voting System for the Olympic Games
}

\author{
Ramon Schalleck \\ Ludwig-Maximilians-Universität München \\ Pfeuferstrasse 33, \\ D-81373 München \\ ramon.schalleck@stud.uni-muenchen.de \\ +498974791821
}

\author{
Marcin Bober \\ Warsaw School of Social Psychology \\ os. Piłsudskiego 11/43, 28100 Busko Zdrój \\ mbober@spik.swps.edu.pl \\ +48691972919
}

\author{
Heiko Drewes \\ Ludwig-Maximilians-Universität München \\ Feilitzschstr. 19, \\ D-80802 München \\ heiko@terms.de \\ +4989332339
}

\begin{abstract}
In this paper we describe an audience voting system which can be used for all kinds of judged sport events like diving, synchronised swimming, gymnastics, and ice-skating. The basis of the system is cameras, which are fixed to the ceiling. Each camera can cover approximately 1000 spectators of the audience. The image processing software recognises the judging, displayed by boards from every individual spectator. The cost of the solution is quite low, because we need less than 20 cameras for an audience of up to 15,000 spectators and you can use them for surveillance purposes, too.
\end{abstract}

\section{Author Keywords}

Audience voting system, image processing, Olympic Games, judging board

\section{ACM Classification Keywords}

H 5.2 User Interfaces, User-centered design, Input devices and strategies.

\section{INTRODUCTION}

Involving the audience has been of great interest in advertising and TV production over many years. New technologies to measure the response of the audience are central in this field [1]. However the technology is either integrated into certain devices (e.g. a TV) or dedicated systems are designed for relatively small numbers of participants and fairly expensive (e.g. 250 people at a hire rate of 5000\$/day, see [2]).

\section{Design Goals}

Our design aims at creating a system where several thousands spectators can take part in voting. The following considerations are regarded as central for the design.

- Inclusiveness. Every spectator should be able to participate. We like to follow the concept: one person one vote.

Copyright is held by the author/owner(s). CHI 2004, April 24-29, 2004, Vienna, Austria. ACM 1-58113-703-6/04/0004.
- $\quad$ Ease of use and accessibility. Spectators should be able to use the system immediately independent of their background. This should include usage by people with disabilities.

- Robustness. The system should be simple and robust with regard to installation and maintenance.

- Cost efficiency. Development, installation, and operation of the system should be fairly cheap.

- Sustainability. The system should be designed in a way that it can be used in further events and that it is ecological.

\section{Discussion of Design Alternatives}

For TV shows it is quite common to measure the volume of the applause (applause meter) with microphones. This method - although entertaining for the audience - rests to be quite inaccurate when thinking of audiences which support their favourite athletes with electrically amplified noise generators like megaphones or horns. Other approaches like call polls where TV viewers can vote by dialling a certain number are suited for a large audience but not for people at the place of the event, as long as not everyone is equipped with a mobile phone.

In parliament every seat has an input device for taking a vote. Because audiences in a sport arena are much larger (6000 or 15000 people), the installation of such a voting system would be much too expensive and technically complicated. Also theses systems are susceptible to vandalism.

With the spreading of mobile phones and SMS, it is a nice idea to use such devices for a voting system. This could be the voting system for the future, but at the moment there are too many different mobile communication standards, no proof, that somebody from outside (in front of the TV) can vote, and the question who will carry the cost for the communication. The system is not tamper proof either, because it is unlikely that every spectator has a cell phone. And what if someone brought more than one? A solution with mobile phones needs the involvement of the big mobile phone manufactures and provider companies. 
Unless one company will sponsor all, it will take a while, until there is an inter-company solution.

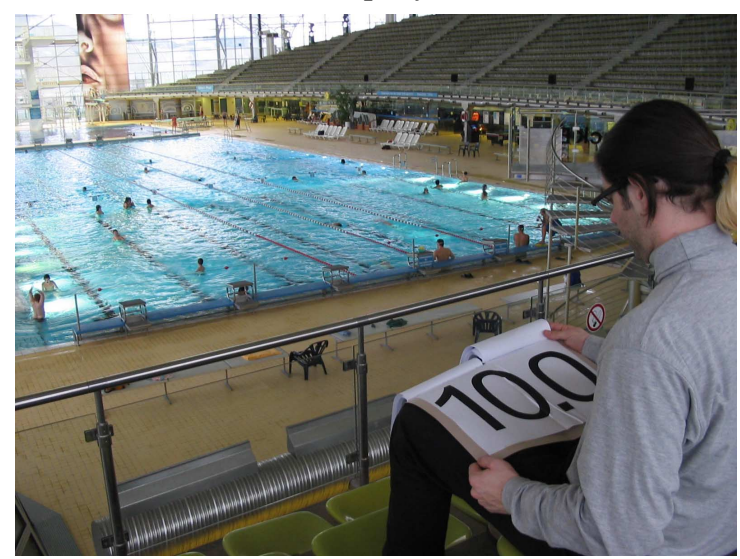

Figure 1: spectator with judging board in the Olympic swimming hall in Munich

A further approach is to facilitate paper and computer vision as input mechanism. Wellner explores this in the context of offices applications [3]. Paper is a cheap medium and its utilization comes natural to all people. It seems also feasible to make the paper used for input part of a brochure people get anyway. In other areas the use of paper for interacting with digital systems has received some attention in research; examples are outlined in "The Myth of the Paperless Office" [4].

With regard to the design goals stated earlier we have come to the conclusion that the use of paper and cameras in conjunction with image processing software bear the most advantages. Both cameras and image processing software are well developed and cheap to get.

\section{Scenarios and Concept}

With the following scenario we like to give the reader an impression how we envisage the participant experience in taking part.

\section{Scenario}

A group of friends buys tickets for a synchronised swimming competition. With their tickets they get a booklet that contains information about the location of the event, the time when competitions starts, information on public transport, a brief history of this sport discipline, a set of pages with numbers, and of course advertisements from the sponsors. The booklet is spiral bound.

They go to the location in time, for the event they are interested in, find their seats, and have a great time seeing the athletic performance.

At the end of each performance the moderator talks during the time the judges need to make up their mind and give their judgement. The group of friends (as every other spectator in the audience) is asked by the moderator how they feel about the performance and to give a judgment by using the numbers in their booklet. They are advised to put it on their knees or to hold it over their heads.
Shortly after that, the moderator announces the final judgment from the jury - but he also relates it to the votes of the audience.

At first they might be confused but then they realize that there is a system to track their votes. For the next performances they are even more excited since they can actively take part.

\section{Concept}

Our system will be installed in a large stadium and its utilization works as follows:

When the audience sits down at the swimming stadium each spectator is equipped with the judging board that lets them combine the numbers for their vote (see Figure 1).

The audience interacts with the stadium display and the moderator when the audience casts its vote which happens when the jury casts its vote, too. As a support for the audience a moderator gives the signal to vote. This can be enhanced by the utilization of a clicking sound, the display, a visual signal like a flash, and a countdown.

In the acquisition phase all cameras simultaneously take one picture per second in a 10 second time window after the signal. The 10 second window is used for verification of the data.

After a few seconds the moderator presents the result of the vote and it is shown on the stadium display.

\section{System Architecture}

Basically the audience voting system consists of cameras, computers and paper. The cameras send the image to a computer. The computer processes the image and calculates the result.

Each camera is attached via USB to one computer (potentially an industrial embedded computer mounted close to the camera). Dependent on the location and the architecture of the building it may also be possible to wire two cameras (observing adjunct blocks) to one PC. These computers capture the image, do the processing and the OCR (optical character recognition) and provide results so that they can be remotely accessed (e.g. by HTTP). The computers are networked, again depended on the building this may be realized using a wired $100 \mathrm{Mbit} / \mathrm{s}$ Ethernet connection or a wireless $54 \mathrm{Mbit} / \mathrm{s} \mathrm{WiFi}$ network. The system requirements for the computers doing the processing are fairly low.

From one central computer all those computers can be accessed and it computes the overall result. Using distributed computers close to the cameras seems to be a more robust solution than connecting all cameras to a single machine. Also networking the cameras over a longer distance would require special hardware. The selected solution is also more reliable because if one combination of camera and computer fails, the others still work and compute their results. There are more advantages; since the 
results of the individual computers can be accessed remotely more than one central computer can be used for different purposes. That way we can provide a computer for the moderator, one for the stadium display, one for the press centre, one for the Olympic Games web page, and one for a social science network.

\section{The Camera}

The Camera should be fixed to the ceiling, so the pictures it takes are bird's views. In a stadium like the Olympic Indoor Hall at the Athens Olympic Sports Complex which has a seating capacity of 15,000[5] and where Artistic Gymnastics and Trampoline competitions will be held, the seats are grouped in blocks, so one camera should cover one block. The resolution of the camera should be high for good recognition results. For image acquisition a still image camera (SLR) with at least 6 Mega Pixel will be used. The camera is connected to computer by USB or Firewire. Using this connection the camera is controlled and the images are accessed. The use of an SLR allows to attach lenses to suit the particular setting (distance from mounting point to audience, light conditions, etc.). With current we would use a Canon 10D camera [6] connected via USB and controlled using the remote capturing software.

So the data for one picture has a size of 9-20 megabytes depending on the compression level and with a cheap 100 megabit/second Ethernet more than one picture per second can be transferred. If the cameras should be used for other purposes, for example as surveillance cameras, a motion picture camera may be preferable. If the motion pictures have a lower resolution, just taking more cameras can easily modify the concept. With a 3-mega-pixel camera and a block of 1000 seats, every seat gets 3000 pixel, which means a cell size of $60 \times 50$. This size is sufficient for character recognition on a board, which lies on the spectator's knees. With a 6 mega pixel camera like the Canon 10D 2000 seats can be covered or the resolution for 1000 seats is doubled.

\section{The Grid}

To recognize the vote of each spectator a grid is placed over the photo. Each square covers one seat and only one vote per seat is counted. That way tampering with the system is reduced to a minimum.

Because the camera will not be fixed exactly above the centre of a block of seats, there will be some perspective distortion. The first step is a geometric correction, so that every seat gets a rectangular piece of the picture (Figure 2). The rectangular pieces can be given separately to recognition software.

\section{The Recognition Process}

When the system has made the photo of the audience, the imaging software will cut out every square of the above mentioned grid. As a result there will be 15000 single images.
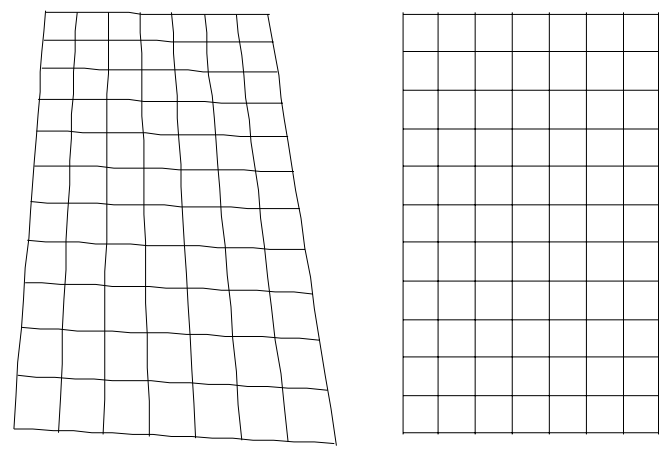

Figure 2: Geometric correction

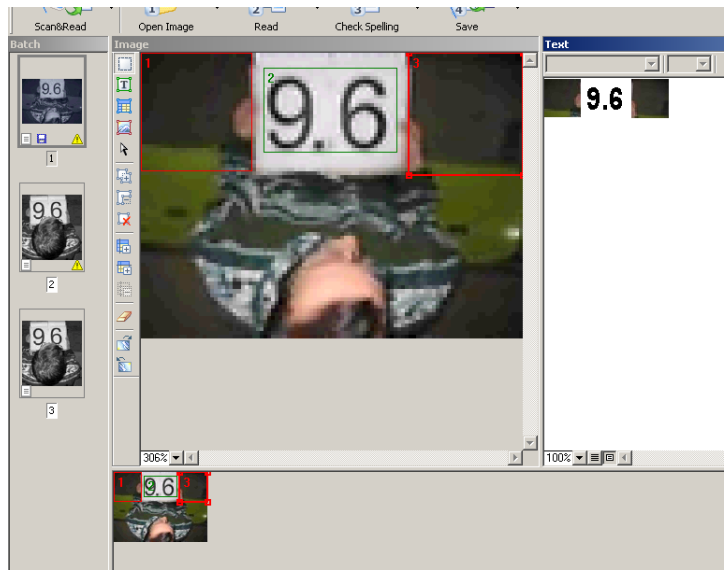

Figure 3: recognition process

With a batching process OCR software like ABBYY FineReader OCR Professional 7.0 converts the images to numbers which are saved in text files (Figure 3). A Perl program is used to further collect the numbers from the files and to compute the average of all numbers. The result will be a digital value which can be processed further in any desired way.

\section{The Spectator's Judging Board}

It would be nice, especially for ecological reasons, if the spectators could give their judgement without any extra material. For a more fun related use of the system it would be enough to detect the motion of clapping hands. This could be done easily by just taking the difference of successive pictures.

For a more serious application, like giving marks as two digit values as it is done in gymnastics and diving an extra device is needed.

One easy approach to solve this problem is to hand out 21 sheets of paper to every spectator. On the papers are just two sets of digits from 0 to 9 and one with 10.0. To avoid mirror confusion for example 6.6 with 9.9 the numbers have a dot at the bottom which indicates the right position of the numbers.

The sets of paper are cheap to produce and can be integrated in a brochure. The backsides of the sheets are free for further information about the sport disciplines, a calendar with the events, maps of the stadium, sponsors 
messages, and a manual for the voting system. The papers must be individually fixed to the brochure cover at the top next to each other and the 10.0 spans two single papers to prevent the score of 10.1-10.9 points (Figure 4).

Since a small amount of guidance will be needed to use the voting board, there should be a person who gives the commands when the audience has to cast the vote. To let the audience know when their participation is being recognized the capturing of the judgment can be indicated in some way. Since we use cameras for the capturing it is plausible to use a clicking sound and a flash. Each time a vote is being captured a bright flash and a click over each block is initiated. When asked to give the judgement, a spectator just has to combine the two sheets with digits in his book and put it on his knees. It is essential for the camera to pick up the numbers that the audience sits strait. After taking 10 pictures the images can be processed.

\section{Recognition problems and Tampering}

The system should be tamper proof. Every person should only have one vote. Unverifiable votes do not count. We have dealt with this problem by placing a grid over the image. Every square covers the space of one seat.

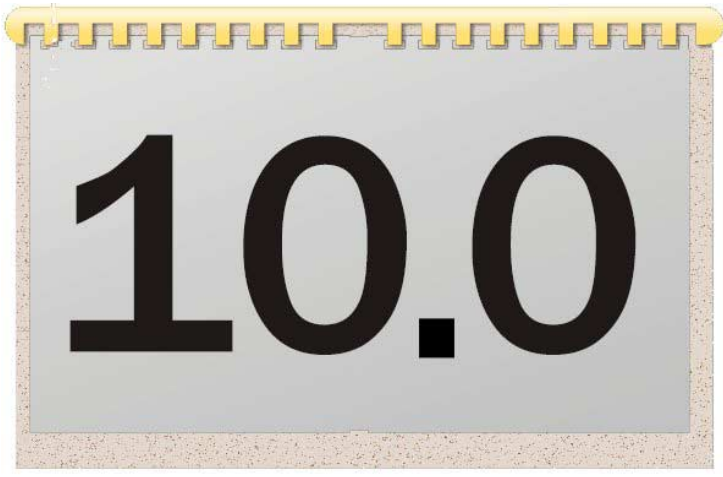

Figure 4: audience judging board

\section{Displaying Results}

Every sports stadium has a big display board. So it is the cheapest way to use this board for displaying the results of the audience votes. Nowadays such display boards consist of controllable pixels, and not of letters (as known from airports).

This display can show the grid of the seats like a mirror. Every cell of the grid corresponds to a seat and shows the judgement detected for this seat. So every spectator can identify himself on the display and verify the mark he gave. The normal display of points in a competition can be enriched by the audience votes, too. The result of the audience vote can be written next to the final result of the official judges to compare them.

Figure 5 shows the grid with seats. In every rectangle there is the number that was captured from the seat. Since there are thousands of seats the display shows one block after the other before the final result is presented by the moderator and is shown on the display.

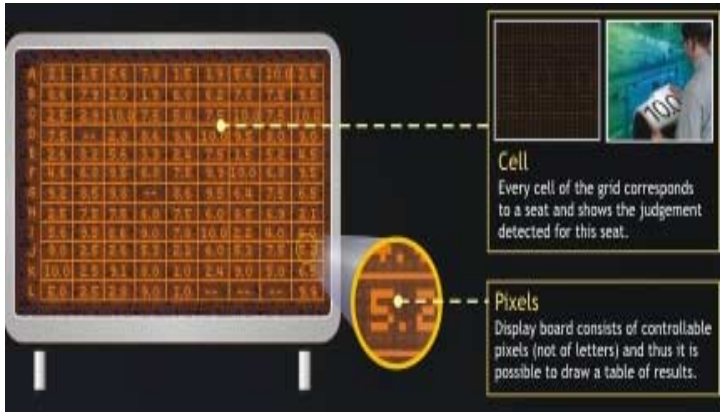

Figure 5: output of audience votes on stadium display

\section{Moderator}

The output of the audience voting system functions partly with a human moderator. He controls the output and can be used to increase the excitement in the audience.

The moderator gives the instructions for the voting with a countdown. He pronounces the result of the vote in addition to the visual result on a screen and comments it.

\section{Costs}

15 x 3 mega pixel digital camera $(\sim 300 \$ /$ piece $)$

16 x PC ( 500\$/piece)

Imaging software is rather easy to develop and there are a lot of open source projects which deliver optimal results in these areas (Photoshop alternatives like Gimp).

\section{CONCLUSION}

The given solution is cheap and flexible.

\section{ACKNOWLEDGMENTS}

We thank our teacher Albrecht Schmidt, who gave us the idea to participate in this competition.

\section{REFERENCES}

[1] Friea Crocker (1996). Trends and Developments in MomentTo-Moment Research using Electronic Group Measurement Systems. International Conference on Computer Assisted Survey Information Collection, December 1996, San Antonio, TX, USA. http://www.ms-interactive.com/docs/history.pdf

[2] Audience Voting Inc.

http://www.audiencevoting.com/ visited 18.12.2003

[3] Wellner, Pierre D. (1993) Interacting with paper on the DigitalDesk, Communications of the ACM Volume 36 No 7 pp87-96, July 1993.

[4] Abigail J. Sellen, Richard H. R. Harper (2001). The Myth of the Paperless Office. MIT Press.

[5] Olympic Games, Athens 2004

http://www.athens2004.com/athens2004/page/venues?lang=en\&ci $\mathrm{d}=$ fa1a470429149f00VgnVCMServer28130b0aRCRD\&oid=0e29 f194d0169f00VgnVCMServer28130b0aRCRD visited 7.1.2004

[6] Canon EOS 10D specification. 2004.

http://www.canoneos.com/ 